Military Technical College

Kobry Elkobbah,

Cairo, Egypt

May 27-29,2008

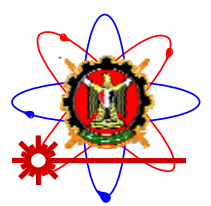

$4^{\text {th }}$ International Conference on Mathematics and Engineering Physics (ICMEP-4)

PH-7

\title{
Simulation and Analysis of a High Power Diode-End-Pumped Nd:YVO 4 Solid- State Laser
}

\author{
Ahmed.M.Samy ${ }^{1}$, Ashraf.F.El-Sherif ${ }^{2}$, Ayman.M.Mokhtar ${ }^{3}$, Mahmoud.F.M.Hassan ${ }^{4}$
}

\begin{abstract}
$\mathrm{Nd}: \mathrm{YVO}_{4}$ is widely and commercially available laser material for diode-pumped lasers. Compared to $\mathrm{Nd}: \mathrm{YAG}, \mathrm{Nd}: \mathrm{YVO}_{4}$ has a larger stimulated emission cross-section, larger absorption coefficient and broader absorption bandwidth. It has lower threshold and its output power is less sensitive to the drifting of the diode pump wavelength. In this paper a Nd:YVO 4 crystal at $1064 \mathrm{~nm}$ pumped with a high power diode laser at $808 \mathrm{~nm}$ is modeled and simulated using LasCAD software tool package. These models investigate the optimum cavity design parameters (length of the cavity, output coupler reflectivity, the beam overlap key parameter and crystal dimensions). The geometry of the cavity is optimized to produce maximum $\mathrm{TEM}_{00}$ laser output, taking into consideration the thermal gradient on the rod structure. The cavity optimum configuration neglecting the insignificant effect of changing rod length was; $66 \mathrm{~mm}$ in length, $90 \%$ of output coupler reflectivity. The pumping beam spot size affects on the laser output and the final optical conversion efficiency. Controlling the pumped spot size $\left(w_{g}\right)$ to be equal the mode spot size $\left(w_{B}\right)$ the maximum beam overlap efficiency and consequently the maximum optical slope efficiency with the highest laser output were achieved. The Nd:YVO $\mathrm{Crystal}_{4}$ with dimension of $(1-3 \mathrm{~mm})$ diameter and $(7-10 \mathrm{~mm})$ length with $1 \%$ doping concentration (1at.\%) is the suitable choice for the DEPSS (Diode End Pumped Solid State ) laser configuration. A final optical conversion efficiency of 52\% and a maximum slope efficiency of 54\% were obtained. These results can be improved by rescaling the transfer efficiency to become around $90 \%$.
\end{abstract}

\section{1- Introduction :}

Three decades of solid-state laser research have contributed to the development of a unique family of powerful photonic tools. Solid-state laser media, such as neodymium-doped crystal, is used in applications like laser fusion, material processing, optical communications, product marking, remote sensing and surgery [1-2].

In recent years; development in high power diode pumping contributed in a significant advancement in solid-state laser design, improvement and applicability. Optical pumping using a semiconductor laser diode instead of conventional flashlamp provides more efficient, reliable, stable laser $\mathrm{o} / \mathrm{p}$ and compact all solid-state laser sources. In contrast to flashlamp pumping (the conventional method for generating a population inversion in solid-state gain media); excitation using a monochromatic and spatially coherent laser pump source offers lower threshold, higher o/p efficiency, reduction of thermal loading and improving the mode quality.

At the time; diode pumping was first proposed, semiconductor lasers were in their formative years, providing low powers (a few milliwatts); they were not competitive with flashlamps, until efficient high power AlGaAs laser diode became available in the mid-1980 [3-4]. Today; reliable one and two- 
dimensional laser diode arrays are commercially available with continuous-wave and quasi-cw peak powers up to $100 \mathrm{~W}$ and $10 \mathrm{~kW}$ respectively [5].

The output spectra of the first high power diodes are well matched to the absorption peak of neodymium ion $(2 \times 800 \mathrm{~mm})$ [4]. Development of these laser diode pump sources is ongoing with increasing output powers, increasing brightness and new wavelengths.

Simulation using LasCAD tool package provides complex engineering tools; developed on purpose for ease-of-operation. The graphical user interface of the program can be used like an optical work-bench on the PC, allowing intuitive design of laser resonators. In this way LasCAD helps users in laboratories and workshops process experimental results without wasting valuable time.

In this paper; a performance modeling of a diode single end pumped $\mathrm{Nd}: \mathrm{YVO}_{4}$ laser is presented in section (2); to investigate the dependency and inter-relation between the various design parameters. In section (3) the simulation tool was overviewed and the DPSS laser system setup was shown. In section (4) the optimization of the DPSS $\left(\mathrm{Nd}: \mathrm{YVO}_{4}\right)$ Laser system design is discussed; these included; optimizing cavity design, optimizing system design by pumped beam waist size controlling and finally optimizing $\mathrm{Nd}: \mathrm{YVO}_{4}$ crystal dimension. In section (5) a discussion and results for the temperature effect on the $\mathrm{Nd}: \mathrm{YVO}_{4}$ crystal is simulated.

\section{2- Performance modeling of a Diode End pumped Nd:YVO 4 laser :}

In this section a performance model for Diode End Pumped Solid State (DEPSS) Nd:YVO laser will be presented. That model will lead to a good estimate for the dependency and inter-relation between the various design parameters. This will help in optimization of the overall laser efficiency (maximizes system efficiency).

\section{Nd: $\mathrm{YVO}_{4}$ crystal parameters optimization :}

For a high power-diode-pumped $\mathrm{Nd}: \mathrm{YVO}_{4}$ laser ; the relation between the output optical power (laser out from the DPSS system) and the input one (laser from the diode) can be described as [2], [6-7]

$$
\begin{aligned}
& R_{\text {out }}=\sigma_{\text {optical }} \times P_{\text {hn }}
\end{aligned}
$$

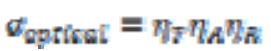

Where $P_{\text {ous }}$ is the laser output power at $1064 \mathrm{~nm}, P_{\text {bn }}$ is the diode laser input power at $808 \mathrm{~nm}, \sigma_{\text {optical }}$ is the optical slope efficiency given by eqn. (2.2); as $\eta_{T}$ is the transfer efficiency, $\eta_{A}$ is the total absorption efficiency and $\eta_{R}$ is the resonator efficiency.

The transfer efficiency is defined as the fraction of the laser pumping radiation leaving the laser diode source and intersects with the laser rod.

$$
\eta_{T}={ }^{P_{T}}{ }_{P_{\mathrm{m}}}
$$

Where $\mathrm{P}_{\mathrm{T}}$ is the fraction of this radiation transferred into the laser active medium. Transfer efficiency can be controlled to get maximum efficiency by the design issues based on the geometrical shape of the pump cavity, these included; the rod diameter and separation of the pump source and laser rod, reflectivity of the laser rod's input face and finally the reflection losses at the coolant jacket.

In the case of end pumping solid state laser the radiation transfer is much simpler as the transfer system consists of antireflection lenses which collimating and focusing the pumping radiation to the laser crystal. Thus we can define the transfer efficiency in terms of reflection losses over all optics and active medium represented by the parameter $(\tau)$.

$\eta_{T}=(1-r)$

Since in our case the laser crystal and optical components are all anti-reflection coated and the radiation transfer losses are very small $(r=5 \sim 20 \%)$ [6-7].

The absorption of pumped radiation by the solid state active medium represented by $\eta_{A}$ is defined as

$$
\eta_{A}=\eta_{a} \times \eta_{Q} \times \eta_{s}
$$

Where

$$
\eta_{\mathrm{s}}=1-\exp (-\alpha L)
$$


$\eta_{s}=\frac{\lambda_{\mathrm{y}}}{\lambda_{E}}$

Where $\alpha$ the absorption coefficient of the Nd:YVO 4 crystal, $L$ is the path length of the gain medium, $\eta_{Q}$ is the quantum efficiency and $\eta_{S}$ is the stokes shift factor (the ratio of the photon energy emitted at the laser transition to the energy of the pumped photons) and is given by eqn. (7); $\lambda_{P}$ is the pumped laser wavelength $(808 \mathrm{~nm})$ and $\lambda_{g}$ is the emitted laser wavelength $(1064 \mathrm{~nm})$.

Equation (6) is a good approximating formula that describes the relation between the absorption efficiency and the absorbed coefficient of the solid state active medium [7-8]. The higher absorbed coefficient makes it easier to obtain single longitudinal mode operation by using a short monolithic cavity and lead to a higher slope efficiency for the $\mathrm{TEM}_{00}$ mode [9].

The final efficient design parameter $\eta_{B}$ was obtained by the multiplication of the two factors; the beam overlap efficiency $\left(\eta_{B}\right)$ and the coupling efficiency $\left(n_{C}\right)$.

Where $n_{B}$ describes the spatial overlap between the resonator modes and the gain distribution in the gain medium, so in case of End-pumped laser; where the output beam from the diode laser is circulated and focused onto the gain medium; the perfect beam overlap is achieved.

The beam overlap efficiency can be expressed in terms of the beam profile spot size $\left(w_{E}\right)$ and the pumped waist spot size $\left(w_{g}\right)[1],[7],[10]$.

$\eta_{B}=\frac{2 w_{E}^{2}}{w_{E}^{2}+w_{g}^{2}}$ for $w_{g}>w_{E}$

And

$$
\eta_{E}=1 \quad \text { for } w_{g} \leq w_{B}^{v}
$$

Finally; the coupling efficiency is being considered as an indication to the reduction of the available output power due to losses in the resonator [2],[7].

$$
\eta_{c}=\frac{T}{(l+T)}
$$

Where $l$ is the round trip losses factor and $T$ is the output coupler transmission factor.

\section{The $808 \mathrm{~nm}$ pumped $\mathrm{Nd}^{3+}: \mathrm{YVO}_{4}$ modeling result :}

A discussion for the end pumping solid state laser configuration advantages was clarified and the calculation for the overall Optical system efficiency according to the previous performance model were done. As the radiation from the high power laser diode module is focused on a small spot on the end of the Nd: $\mathrm{YVO}_{4}$ laser rod with a suitable antireflection coated focusing optics. The laser diode module pump radiation can be adjusted to be coincident with the diameter of the resonator modes to produce a prefect overlap. The key parameter that determines the laser efficiency and the output power is the spatial overlap; it will be shown in section (4.II) that the size of the pump distribution with respect to size of the Gaussian laser mode is usually the most important factor that determines the TEM $_{00}$ resonator mode and the maximum $\mathrm{o} / \mathrm{p}$ power.

The end pumping configuration allows the maximum use of the energy from the laser diode; this configuration is the best manner of operation in producing fundamental spatial mode without entracavity apertures [7],[10].

In this work we will consider the configuration illustrated in figure (1).

Figure (1) is a Plano-concave resonator configuration with an anti-reflection coated i/p mirror at $808 \mathrm{~nm}$ and high reflection coated at $1064 \mathrm{~nm}$ and an o/p coupler with partially reflected coated at $1064 \mathrm{~nm}$. 


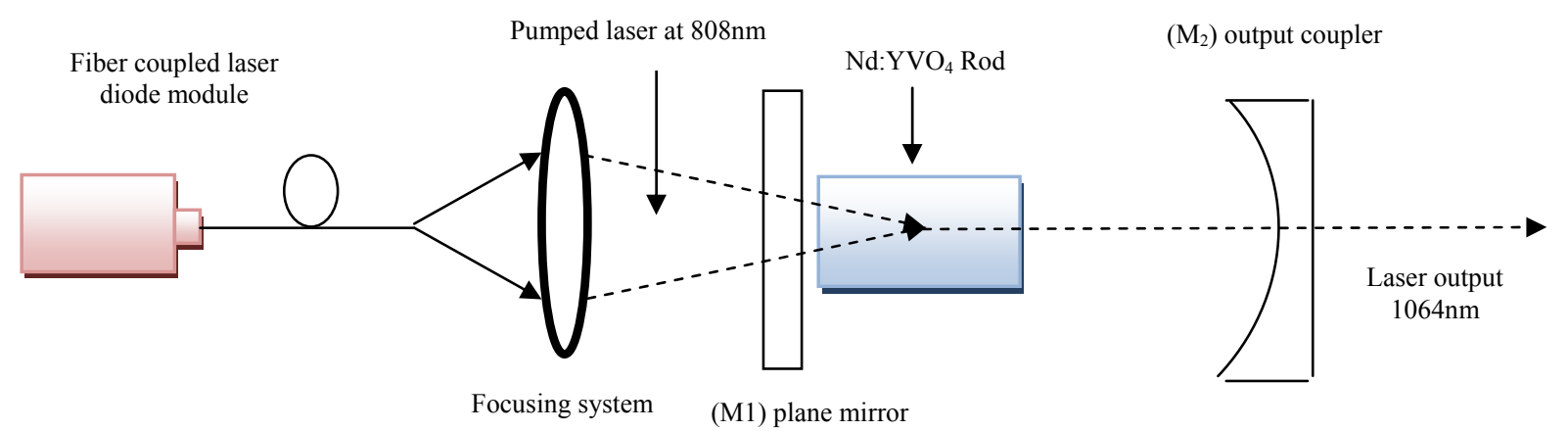

Figure (1) the DPSS Nd:YVO 4 laser with the a single End pumping configuration

The output from the laser diode module with a maximum of $7.8 \mathrm{cw}$ watt at $808 \mathrm{~nm}$ is collimated and focused onto $3 \mathrm{~mm}$ diameter $\times 10 \mathrm{~mm}$ long of $\mathrm{Nd}: \mathrm{YVO}_{4}$ laser Rod with 1 at.\%.

The transfer efficiency $\eta_{T}$ will be around 80-98\% [2],[6-7] depending on the anti-reflection coating on the $i / p$ mirror and the distance between the diode and the gain medium. The pump radiation will be absorbed according to the totally absorbed efficiency that determined before, where the absorption coefficient factor for the $\mathrm{Nd}: \mathrm{YVO}_{4}(1 \mathrm{at} . \%)$ crystal is equal to $31.4 \mathrm{~cm}^{-1}$ [11], $\eta_{Q}=0.95$ [7][11], $\eta_{s}=0.759$ producing total absorbed efficiency $\eta_{A}=0.72$. By evaluating the beam overlap efficiency $\eta_{B}$ in eqn. (9) and calculating $\eta_{C}$ for an o/p coupler of $90 \%$ reflectance at $1064 \mathrm{~nm}$; one can calculated the Optical slope efficiency to be equal $48 \%{ }^{1}$, figure (2) shows the optical to optical power curve calculated through eqn. (1).

The attractive features of $808 \mathrm{~nm}$ end pumped $1064 \mathrm{~nm} \mathrm{Nd}: \mathrm{YVO}_{4}$ is its very compact design, in addition to the high beam quality and efficiency as a result of the good overlap between the pumped region and the $\mathrm{TEM}_{00}$ laser mode.

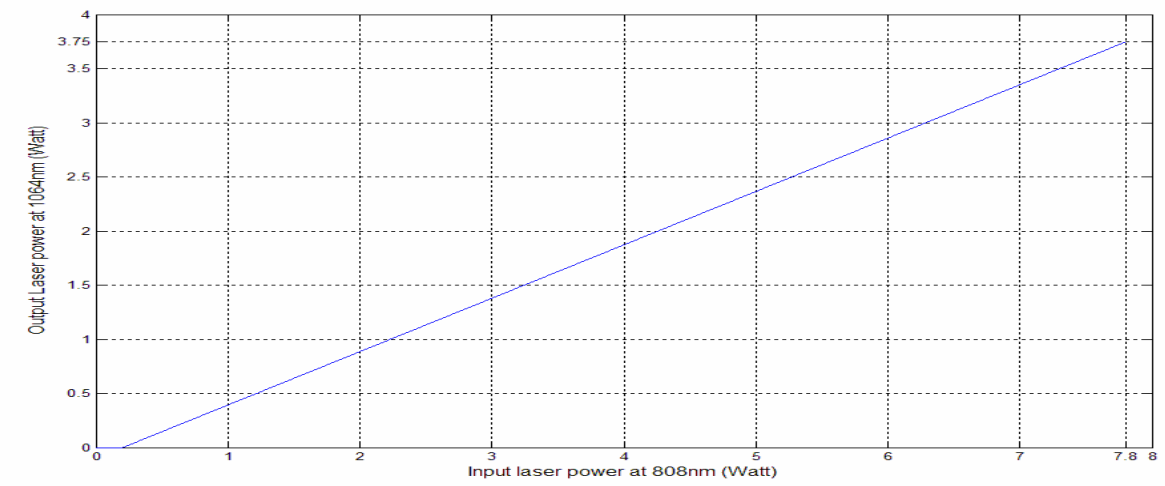

Figure (2) the calculated o/p laser power as a function of $i / p$ laser one.

\section{3- Simulation studies:}

LasCAD software simulating tool package was use to simulate and analysis the setup shown in figure(1). LasCAD provides multiphysics analysis of the complicated interaction between thermal and optical fields in solid state lasers commonly known as thermal lensing effect. Modeling of this effect and its influence on beam quality, cavity stability and laser efficiency is essential for analysis and optimization of laser resonators.

In this section we will discussed the single end pumping configuration as a design issue using solid state $\mathrm{Nd}: \mathrm{YVO}_{4}$ rods with different dimensions and ideal two mirror resonator as shown in figure (1).

${ }^{1}$ We considered the worst case of the transfer efficiency to be $80 \%$ in case of end pumping configuration. 
The Nd: $\mathrm{YVO}_{4}$ crystal was pumped by a high power fiber coupled diode laser module at $808 \mathrm{~nm}$, with a maximum pumped power of $7.8 \mathrm{Watt}$, The pumped beam focused into the laser crystal had spot size of the beam waist around $150 \mu \mathrm{m}$.

$\mathrm{Nd}: \mathrm{YVO}_{4}$ (a-cut) crystal (a1at.\%) was used with different dimensions; to study the effect of:-

a. The rod length on the performing output power.

b. The rod geometrical dimension on the temperature gradients and the catastrophic face damage.

c. The variation of beam waist spot size Vs. output power.

The crystal was AR coated for wavelengths of $808 \mathrm{~nm}$ and $1064 \mathrm{~nm}$; which are the absorbed and output laser wavelengths respectively. The input mirror (M1) was a flat mirror (HR) coated at 1064nm, (HT) coated at $808 \mathrm{~nm}$. The output coupler was (M2) a concave mirror with radius of curvature of $(100 \mathrm{~mm})$; HR at $808 \mathrm{~nm}$ with $10 \%$ transmission factor at $1064 \mathrm{~nm}$.

\section{4- Optimizing DPSS (Nd:YVO $)_{4}$ Laser system : I. Optimizing Cavity design :}

The interaction between thermal effect and optical system denoted by the thermal lens of the laser crystal, it is an important parameter for optimizing the laser system and performing the high power laser operation. When a fiber-coupled diode laser pumped $\mathrm{Nd}: \mathrm{YVO}_{4}$ solid state crystal in $\mathrm{cw}$ mode of operation, the resonator experienced a strong thermal lensing effect with $\mathrm{Nd}: \mathrm{YVO}_{4}$. The thermally induced focusing lens drove the laser resonator out of the stable regime.

In the absence of laser action; the thermal loading on the medium is strong because of the strong heat absorption within the material. Therefore, the thermally induced focusing lens of the material is stronger during non-lasing operation [12-13].

The main point is to determine the pump-induced thermal lens of the $\mathrm{Nd}: \mathrm{YVO}_{4}$ laser medium under lasing condition by using a diode laser pump. After the thermally induced focusing lens was identified, the resonator would be redesigned to achieve a stable laser oscillation. The effective focal length for the entire $\mathrm{Nd}: \mathrm{YVO}_{4}$ crystal is given by [1],[14].

$f=\frac{\pi \kappa_{c} W_{g}^{2}}{P_{p h}(d \pi / d T)}\left(\frac{1}{1-\theta^{(-\infty i)}}\right)$

Where $\mathrm{P}_{\mathrm{ph}}$ is the fraction of the pumped power that results in heating and given by eqn. (12) [1].

$P_{p h}=\left(1-\frac{\lambda_{p}}{\lambda_{E}}\right) P_{m}$

Where $\boldsymbol{F}_{\boldsymbol{m}}$ is the input pumped power, $\lambda_{p}$ and $\lambda_{E}$ are the pumped and emitted laser wavelength as mentioned in section (2), $l$ is the rod length, $w_{g}$ is the spot size of the pumped beam waist (in this case $w_{g}=150 \mu \mathrm{m}$, but we will try a different size to observe the effect on the output power in section(4.II). Table (1) shows the optical and thermal parameters of $\mathrm{Nd}: \mathrm{YVO}_{4} 1.0 \%$ doping concentration (a-cut) lasers. 


\begin{tabular}{|lcccc|}
\hline \multicolumn{1}{|c|}{ Name } & Symbol & Units & Typical Value \\
\hline Atomic Density & $\mathrm{N}$ & Atoms $/ \mathrm{cm}^{3}$ & $\begin{array}{c}1.26 \times 10^{20}(\mathrm{Nd} 1.0 \\
\text { at. } \%)\end{array}$ \\
$\begin{array}{l}\text { Peak Emission Wavelength } \\
\text { Stimulated Emission Cross- }\end{array}$ & $\mathrm{a}$ & $\mathrm{nm}$ & 1064 \\
Section & & $\mathrm{cm}^{2}$ & $25 \times 10^{-19} @ 1064$ \\
Fluorescent lifetime & $\pi$ & $\mu \mathrm{s}$ & 90 \\
Peak Absorption Coefficient & $\alpha$ & $\mathrm{cm}^{-1}$ & $31.4 @ 810$ \\
Refractive Index at 1064nm & $\mathrm{n}_{\mathrm{a}}$ & $\mathrm{K} /(\mathrm{m} . \mathrm{K})$ & 2.17 \\
Thermal Conductivity (300K) & $\mathrm{K}$ & $1 / \mathrm{K}$ & 5.10 \\
Thermal Optical Coefficient & $\mathrm{a}_{\mathrm{s} / \mathrm{d}}$ & $2.9 \times 10^{-6}$ \\
$(300 \mathrm{~K})$ & & & \\
\hline
\end{tabular}

Table (1) characteristics of the optical and thermal properties of $\mathrm{Nd}: \mathrm{YVO}_{4} 1.0 \%$ doping concentration

$$
\text { (a-cut) lasers [11]. }
$$

Applying all these parameters in the first main equation (11); getting the effective focal length for the entire crystal equal to $(6.65 \mathrm{~cm})$. The importance of this result is that the effective focal length of the required pumping rod actually comparable to the resonator length [2]. To verify this condition we simulated different cavity lengths with the same rod of dimension $3 \mathrm{~mm} * 10 \mathrm{~mm}$; with the same output coupler of reflectivity of $90 \%$ at $1064 \mathrm{~nm}$; considering the previous worst case of the transfer efficiency $\eta_{T}$ to be $80 \%{ }^{2}$. The simulation results are summarized in figure (3).

Figure (3) shows the dependence of the output laser power on the designed cavity length; getting the maximum output power $(3.965 \mathrm{~W})$ denoted by solid curve at the cavity length of $66 \mathrm{~mm}$ with an optical conversion efficiency of $51 \%$ and maximum slop efficiency of $(54 \%)$. This result is in a good agreement with the previously calculated one in section (2).

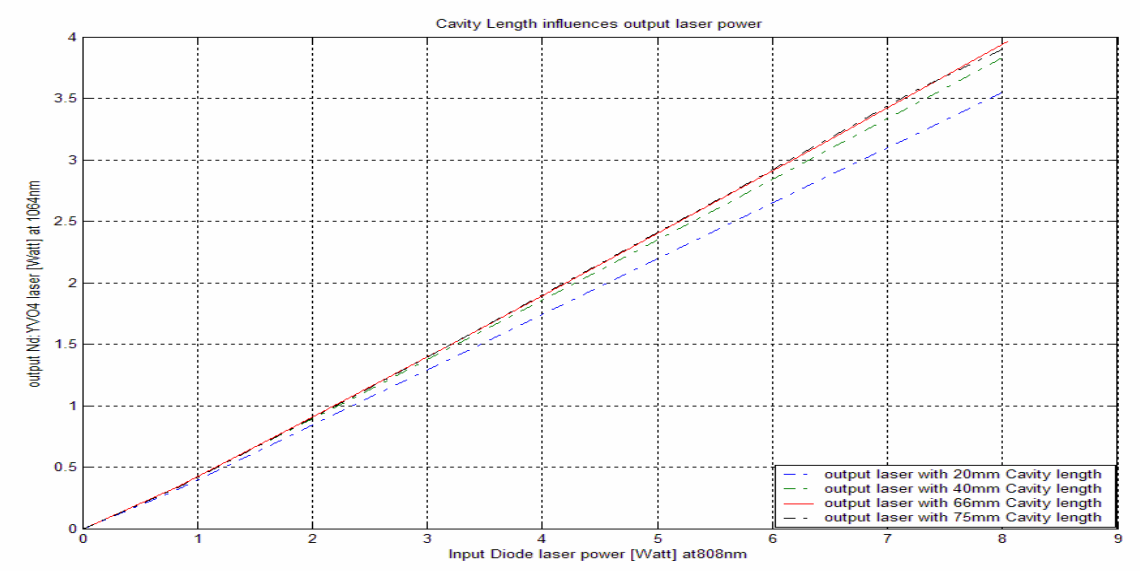

Figure (3) the effect of the cavity length design on the Extract output laser; solid red line was the highest slope efficiency with a maximum extracting laser output up to 3.965 Watt.

We notice that; cavity length above $75 \mathrm{~mm}$ bringing the resonator to unstable condition; figure (4) shows the stability diagram for each simulated case using LasCAD simulating tool package.

\footnotetext{
${ }^{2}$ Transfer efficiency is not an accurate measuring quantity so we toke the worst percentage in case of end pumping system.
} 
II. $\mathbf{O}$

$\mathbf{p}$
$\mathbf{t}$
$\mathbf{i}$
$\mathbf{m}$
$\mathbf{i}$
$\mathbf{z}$
$\mathbf{i}$
$\mathbf{n}$
$\mathbf{g}$

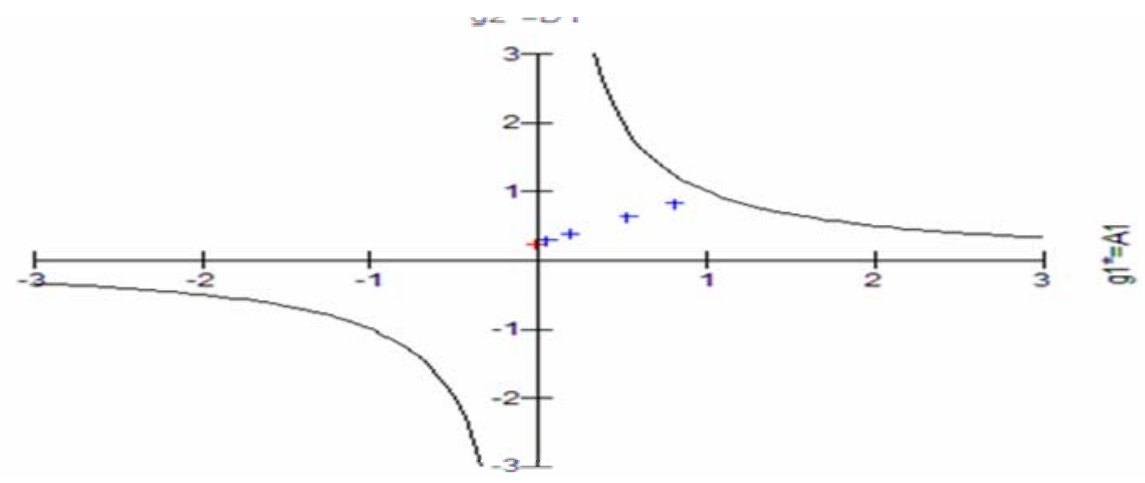

Figure (4) the stability realization of the $\mathrm{Nd}: \mathrm{YVO}_{4}$ designed cavity with $(20,40,66$ and $75 \mathrm{~mm})$ $\mathbf{S}$ in length (from right to left) and shows the unstable case at the cavity total length above $75 \mathrm{~mm}$ $\mathbf{y}$ denoted by the extreme lift cross.

S

tem design by controlling the pumped beam waist :

As mentioned before the resonator length considered to be an important factor in the end pumping design issues. It depends on the square input beam radius of the waist spot size and independent on the rod length.

So the only parameter that can optimize our cavity design is the input beam waist which maximizes the performance of the output laser $(1064 \mathrm{~nm})$ at the given pumped power. As proved before in section (2) the beam overlap efficiency is the most important parameter.

In end Pumping solid state laser controlling $w_{g}$ achieved the benefits of the $\mathrm{TEM}_{00}$ with extracting maximum possible output power. The ratio between $w_{E}$ and $w_{g}$ affects the DPSS laser system performance output, this will be simulated using LasCAD software package.

By simulating a diode end pumped Nd: $\mathrm{YVO}_{4}\left(1\right.$ at.\%) with variation in $w_{g}$ (pumped waist spot size) at the same crystal dimension $3 * 10 \mathrm{~mm}$ and output coupler of $90 \%$ reflectivity at $1064 \mathrm{~nm}$; the importance of this ratio can be shown.

Figure (5) shows the effect of the ratio between the mode spot size $w_{B}$ and the controlled $w_{g}$ on the extracted laser power at $1064 \mathrm{~nm}$.

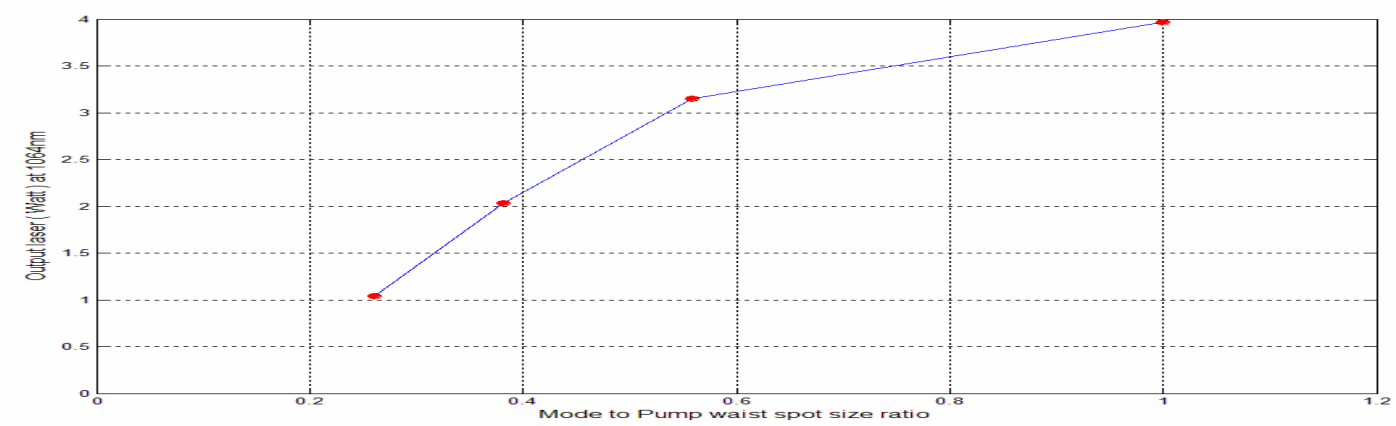

Figure (5) simulated results of the laser output Vs. the mode to pumped beam waist spot size ratio.

From figure (5) and our previous performance analysis work in section (2); the ratio between $w_{E}$ and $w_{g}$ can now show its effect on the system output performance; as the maximum output power $(3.965 \mathrm{~W})$ can be extracted when this ratio exactly equal to $1.0\left(w_{g} \approx w_{g} \approx 150 \mu \mathrm{m}\right)$. As this ratio became smaller than 1.0; the performance laser output would be affected by; and the extracted power will be much less than expected (case of $\eta_{:}<1$ ); this effect is shown at a given $w_{g}=250,350$ and $500 \mu \mathrm{m}$. 
From the above figure the effect of the mode to pump ratio on the output laser power fulfilled the great importance of the beam overlap efficiency.

\section{Optimizing Nd:YVO ${ }_{4}$ Crystal geometry :}

In this work; we will investigate the effect of changing the crystal geometry on the extracting laser o/p. First we simulated $\mathrm{Nd}: \mathrm{YVO}_{4}$ crystal with different lengths.

Figure (6) shows the laser output power (Watt) Vs. different crystal lengths (2, 4, 7, 10 and 15mm). It illustrates the independency of the laser output on changing rod length, moreover; increasing the length of the gain medium will produce insignificant changing in the extracting power; this change is inversely proportional to the rod length.

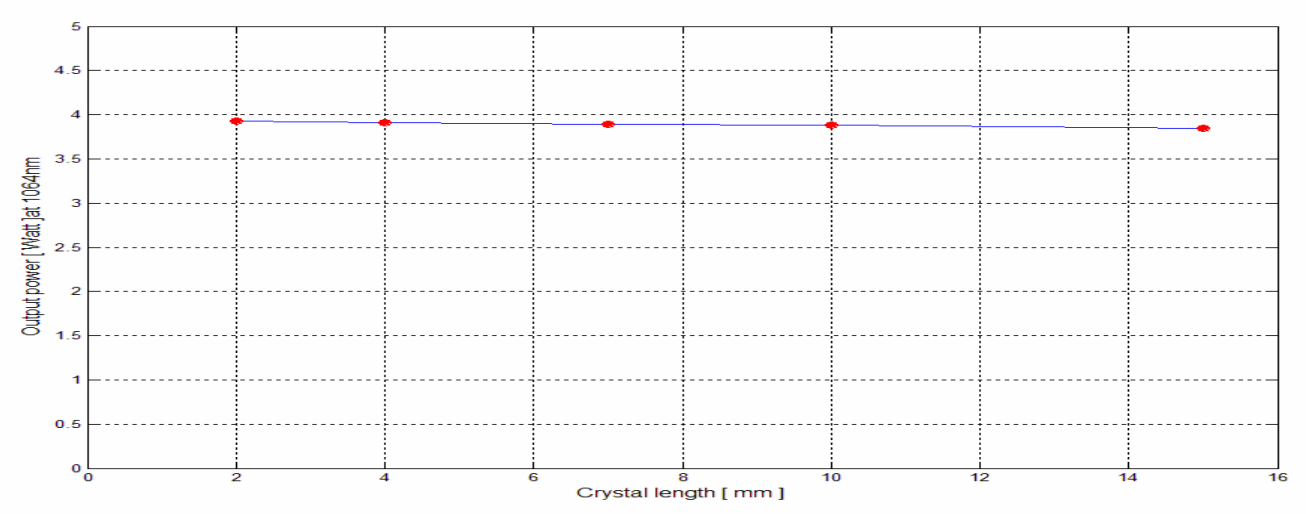

Figure (6) Laser output power Vs. Crystal length curve

As mentioned in [7] and the previous performance analysis in section (2); the total absorption efficiency in case of end pumping solid state laser will be around $72 \%$ of the input $808 \mathrm{~nm}$ laser disregarding to the rod length; so using the previous treatment made only $2 \mathrm{~mm}$ length of $\mathrm{Nd}: \mathrm{YVO}_{4}$ crystal provided laser output power slightly higher than crystal of $15 \mathrm{~mm}$. Figure (6) illustrates the results of this study which was in an excellent agreement with the numerical model of the $\mathrm{Nd}: \mathrm{YVO}_{4}$ laser and its calculated results in[9].

\section{5- Temperature effect on the solid state crystal (thermal fracture):}

From the most recent researches in DPSS lasers and especially in case of end pumping process; progress in scaling the output power while maintaining operation in the $\mathrm{TEM}_{00}$ mode is limited by the formation of an aberrated thermal lens within the active medium [15], moreover; the maximum input pump power is restricted by thermal fracture of laser crystal [16].

Since a large amount of thermal energy converted from the absorbed pump power is accumulated near the pump region of the end pumped geometry; so the temperature distribution inside the laser crystal became gradually more serious as the pump power increases. The maximum output power for DEPSS laser is fundamentally limited by thermal fracture of laser crystals [7]. Moreover; it is so difficult to reduce thermal stress in an end-pumped geometry compared to side-pumped one. Therefore, it is important to simulate the temperature gradient on the front face and inside the pumped $\mathrm{Nd}: \mathrm{YVO}_{4}$ crystal to avoid thermally induced fracture and evaluate the maximum pumped power.

Figure (7) shows the simulated results of two rods of different dimensions $(3 * 2 \mathrm{~mm}$ and $3 * 10 \mathrm{~mm})$. It illustrates the thermal gradient on the front face and inside the $\mathrm{Nd}: \mathrm{YVO}_{4}$ crystal. The results show a central maximum temperature of $312^{\circ} \mathrm{C}$ and $300^{\circ} \mathrm{C}$ for the first and the second rod respectively (other cavity parameters considered to be constant values with $7.8 \mathrm{~W} \mathrm{cw}$ laser input power). 


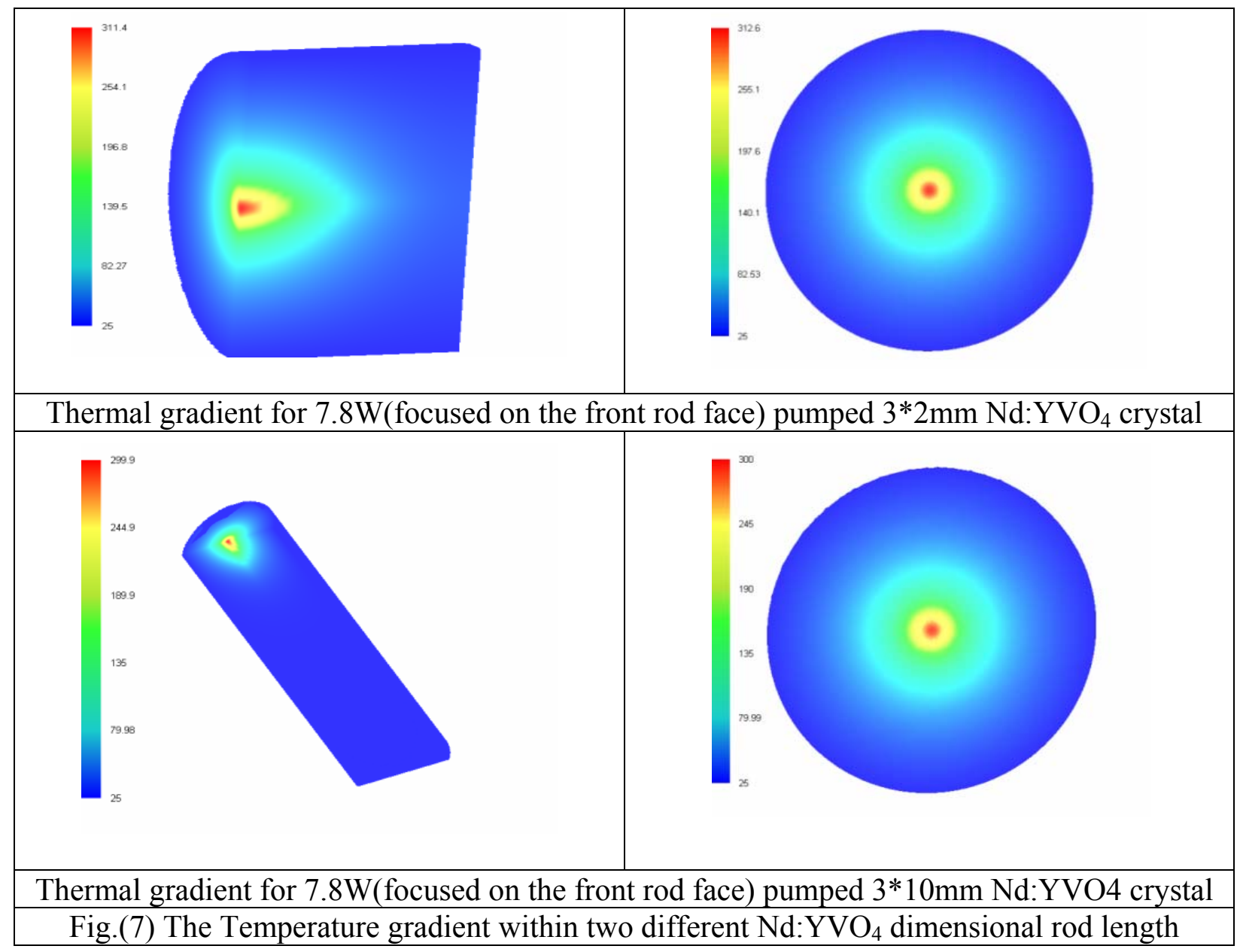

This simulation was deliberated to confirm that; the longer crystal is useful in heat removal, but it increases the intacavity absorption losses as shown in figure (6).

The temperature gradient is also affected by The radial change of the crystal diameter; simulating $\mathrm{Nd}: \mathrm{YVO}_{4}$ with different rod diameter is strongly recommended, figure(8) illustrated this important effect; as the crystal diameter became smaller the minimum storage temperature case is obtained; this is because the deposited heat can be more effectively removed radially from the crystal.

\begin{tabular}{|l|l|l|}
\hline & \\
\hline $\begin{array}{l}\mathrm{Nd}: \mathrm{YVO}_{4} \quad \text { Crystal with dimension of } \\
0.5^{*} 10 \mathrm{~mm}_{2} \text { and central temp. of } 188^{\circ} \mathrm{C}\end{array}$ & $\begin{array}{l}\text { Nd: } \mathrm{YVO}_{4} \text { Crystal with dimension of } 1 * 10 \mathrm{~mm} \\
\text { and central temp. of } 255^{\circ} \mathrm{C}\end{array}$ \\
\hline
\end{tabular}




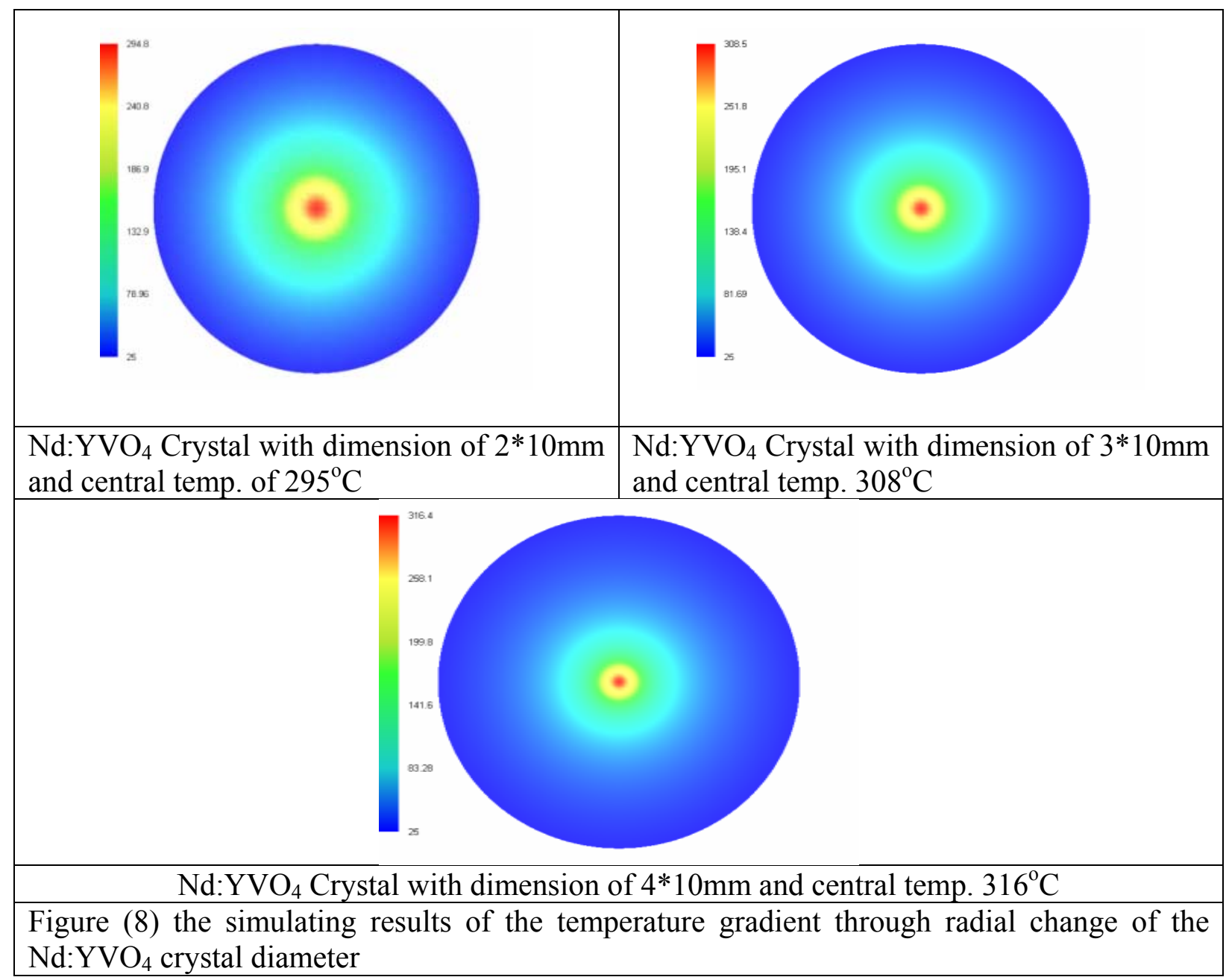

These simulated results manifested the reason of increasing the fracture limit as the crystal diameter became smaller as shown in figure (9).

Figure (9) shows the maximum pumped power (W) Vs. the crystal width (mm); it clarifies that more pumping power can be tolerated with smaller radial diameter.

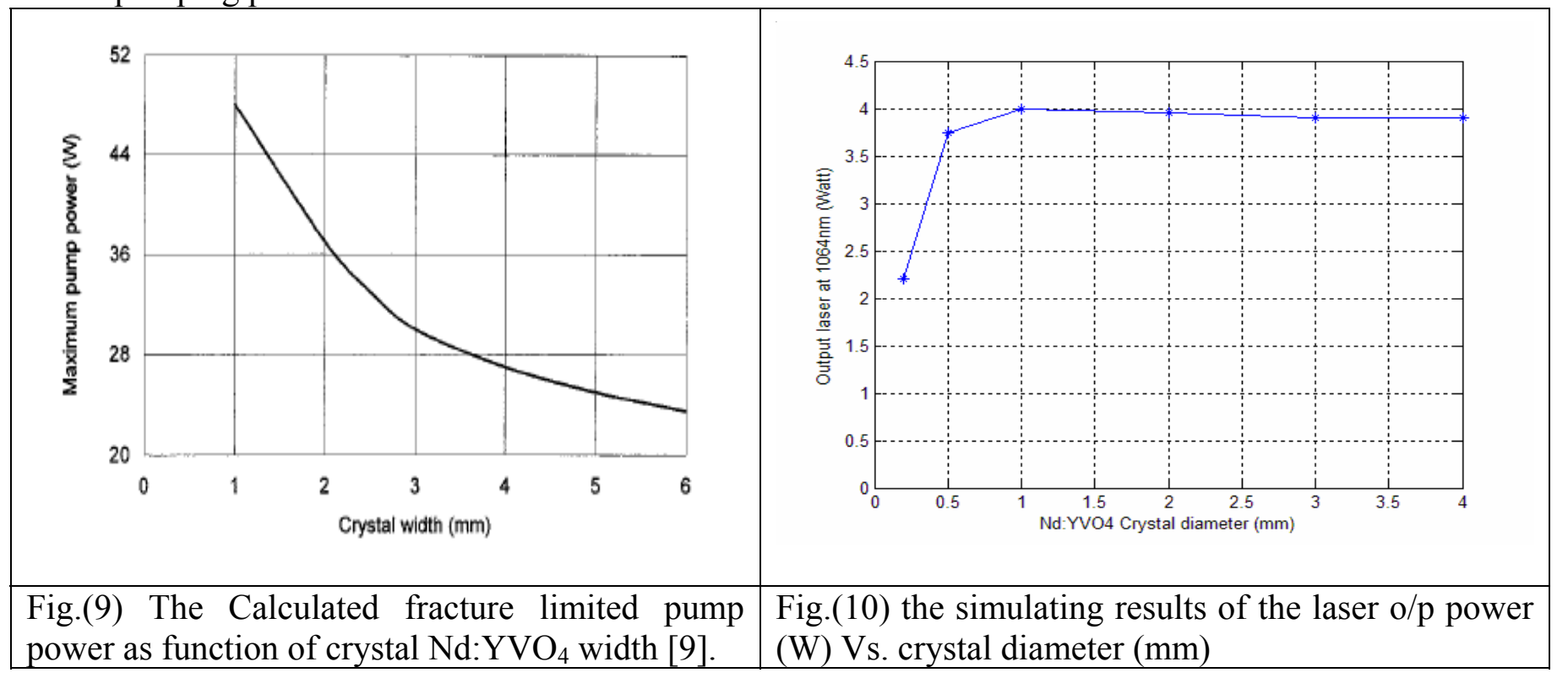


Figure (10) shows the effect of the crystal radial changing on the laser output; these simulated results were in excellent agreement with the numerical solution given by [9].

Figure (10) illustrates that $\mathrm{Nd}: \mathrm{YVO}_{4}$ rod with dimension of $1 * 10 \mathrm{~mm}$ is the suitable choice with 1at.\% of doping percentage; getting maximum output power 4.03 Watt with $52 \%$ of the optical conversion efficiency $^{3}$ and maximum optical to optical slope efficiency of $54 \%$.

\section{6- Conclusion}

The DPSS Nd:YVO 4 Laser was simulated using LasCAD and its performance was analyzed. Nd:YVO crystal with dimension of $(1-3 \mathrm{~mm})$ diameter and $(7-10 \mathrm{~mm})$ length with 1at.\% doping percentage is optimized to achieve the highest $\mathrm{o} / \mathrm{p}$ performance at $1064 \mathrm{~nm}$ (optical slope efficiency). This is done through DEPSS of a plano-concave cavity configuration with an optimized 66mm length. The spot size of the pumping beam was optimized to achieve the maximum o/p power as the mode to pumped spot size ratio equal to $1\left(\mathrm{w}_{\mathrm{B}}=\mathrm{w}_{\mathrm{g}}\right)$ producing maximum beam overlap efficiency and consequently maximum optical to optical slope efficiency with the highest final laser output. The analysis of the thermal gradient on the rod enabled the suitable choice of the crystal dimension.

Final optical conversion efficiency around 52\% and maximum slope efficiency around 54\% were obtained at the transfer efficiency of the pumped beam of $80 \%$.

\section{References}

1. Jimin Yang, Jie Liu, Jingliang He "Efficient diode-pumped Nd:YVO $\mathrm{YV}_{4}$ continuous-wave laser at 1. 34 mm" Optik 115, No. 12 538-540, 2004.

2. Hongrui Zhanga, Mingju Chaoa, Mingyi Gaoa, Liwen Zhanga, Jianquan Yaob "High power diode single-end-pumped Nd: $\mathrm{YVO}_{4}$ laser" Optics \& Laser Technology 35 , 445 - 449 (2003).

3. Govind P. Agrwal (Semiconductor Lasers) second edition.

4. W. Streifer, Donald R. Scifres, Gary L. Hrnagel, David F. Welch, Josef Berger and M. Sakamoto "Advances in Diode Laser Pumps" IEEE J. QE, VOL. 24, NO. 6, 1988.

5. David F. Welch "A Brief History of High-Power Semiconductor Lasers" IEEE J.Q.E, VOL.6, NO. $6,2000$.

6. Oranzio Svleto "principles of Lasers" fourth edition.

7. W.Koechner "solid state laser" fifth edition.

8. Michael bass and J.dong "Properties of diode laser pumps for high power solid state lasers" IEEE J.QE, VOL.41, .2005.

9. Xiaoyuan Peng, Lei Xu, and Anand Asundi" Power Scaling of Diode-Pumped Nd:YVO $\mathrm{YLasers}_{4}$ IEEE J. QE, vol. 38, no.9, 2002.

10. Paolo Laporta "Design Criteria for Mode Size Optimization in Diode-Pumped Solid-state Lasers" IEEE JOURNAL OF QUANTUM ELECTRONICS, VOL. 27. NO. 10, 1991.

11. Crystech Inc. crystals \& optics catalog 2006

12. J. L. Blows, T. Omatu, J. Dawes, H. Pask and M. Tateda "Heat generation in Nd:YVO 4 with and without laser action" IEEE Photonics. Tech Lett., vol. 10, 1998.

13. T. Y. Fan "Heat generation in Nd:YAG and Yb:YAG" IEEE, J.QE, vol. 29, 1993.

14. Innocenzi ME, Yura HT, Fincher CL et al."Thermal modeling of continuous- wave end-pumped solid-state lasers". Appl. Phys. Lett. 56, 1831-1833, 1990

15. K. R. F. Kleine, L. P. Gonzalez, R. Bhatia, L. R. Marshall, and D. G. Matthews, "High brightness $\mathrm{Nd}: \mathrm{YVO}_{4}$ laser for nonlinear optics," in Proc. TOP's Adv. Solid-State Lasers, vol. 26, pp. 271278, 1999.

16. S. C. Tidwell, J. F. Seamans, M. S. Bowers, and A. K. Cousins, "Scaling CW diode-end-pumped Nd:YAG lasers to high average powers," IEEE J. Quantum Electron., vol. 28, pp. 997-1009, Apr. 1992.

\footnotetext{
${ }^{3}$ These results were simulated at $80 \%$ of the transfer efficiency.
} 